\title{
Multibody simulation and control of kinematic systems with FMI/FMU
}

\author{
Francois Chapuis ${ }^{1}$, Jean Daniel Beley ${ }^{2}$, Stephane Garreau $^{3}$, Olivier Roll ${ }^{4}$, Tim Puls ${ }^{5}$, Leon Voss $^{6}$, \\ Sameer $\mathrm{Kher}^{7}$ \\ ${ }^{1}$ ANSYS Inc., France, francois.chapuis@ansys.com \\ ${ }^{2}$ ANSYS Inc., France, jean-daniel.beley@ansys.com \\ ${ }^{3}$ ANSYS Inc., France, stephane.garreau@ansys.com \\ ${ }^{4}$ ANSYS Inc., France, olivier.roll@ ansys.com \\ ${ }^{5}$ ANSYS Inc., Germany, tim.puls@ansys.com \\ ${ }^{6}$ ANSYS Inc., Germany, leon.voss@ ansys.com \\ ${ }^{7}$ ANSYS Inc., USA, sameer.kher@ansys.com
}

\begin{abstract}
Our connected world and environment make us interact every day with very complex devices. Driving our cars, monitoring health using smart phones, the extensive use of robots are all applications which involve large quantities of embedded functions and physics. To design and simulate efficiently such systems, individual physics simulators are not sufficient and coupled simulations are required. A new standard called FMI (Functional Mock-Up Interface) [1] has been created, allowing to federate these interactions between a wide variety of physical, digital and reduced models, either through a co-simulation approach or through model exchange strategy using a standardized and neutral interfacing mechanism. In this article, we illustrate through an example how it's possible to simulate mechanical assemblies, kinematics, dynamics and control systems in the same system model. Each mechanical sub-assembly is represented by a FMU (Functional Mock-Up Unit) exported from a multibody dynamics solver and includes a mix of rigid and flexible components. Flexible components are reduced order models of the full fidelity finite element model using the well-known CMS (Component Mode Synthesis) method. [2]

We apply the coupling through the FMI standard to a robot model, composed of rigid parts and one flexible subassembly. The highly non-linear behavior of the equations of motion of the multibody assembly is captured and consumed as a co-simulation FMU. The actuators detailed model - from the voltage source to the electric motors are modeled in the system simulation platform ANSYS Twin Builder, while the control loops use SCADE which offers different control laws. The co-simulation of these 3 sub-systems can then be performed in an efficient manner, without the prerequisite of having on-off coupling developed between each of the individual simulators.
\end{abstract}

Keywords: FMU, multibody dynamics, CMS, actuator, control

\section{Introduction}

As a prerequisite to a system simulation, it is commonly known that one must build, embed and link all relevant components in a simulation platform. Some of the standard base components are directly available in the Modelica libraries and some other higher fidelity models may also be reduced through ROMs (for instance LTI) or using the FMI standard for model exchange. When the sub-model transient behavior is highly non-linear, no reduction of the model is possible and a co-simulation approach is needed. In that case, a co-simulation FMU is exported, which allows full transient simulation on the fly along with the global simulation. In the shown example, all ROMs and FMUs have been generated from ANSYS Mechanical owing to the finite element and multibody solvers and brought to the systems environment for the whole system simulation.

\section{Generation of the FMUs and the ROM}

The application example is shown in figure 1. It is a 6-axis robot, each of the axis being modeled by a revolute joint on which is added a controlled actuator. 

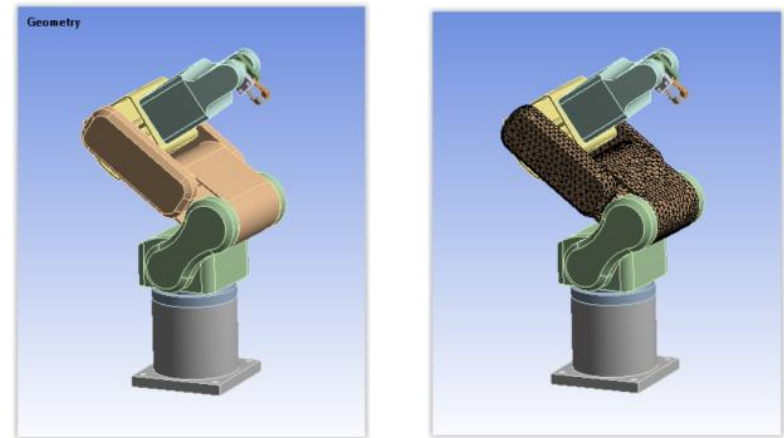

Figure 1 : robot arm geometry and mesh (full rigid on the left, rigid/flexible on the right)

To illustrate multi-level reduced order modeling, one of the arms is considered as deformable. A full fidelity finite element model is built. These 100,000 degrees of freedom model is then reduced to a few dozens of DOFs using a Component Mode Synthesis (CMS) method, accounting for the elastic behavior as well as the coupling between small elastic displacements and large rotations and displacements. A modal analysis of the deformable part, followed by a series of static analysis are performed to generate the CMS reduced order model. During the preprocessing steps, the interface pins are defined. For each robot axis, angle and torque are defined as pins. Finally, we export a co-simulation FMU component (shown in figure 2) with its interface pins. The reduced order model of the flexible part is included in this FMU and will be consumed by the multibody solver during the simulation.

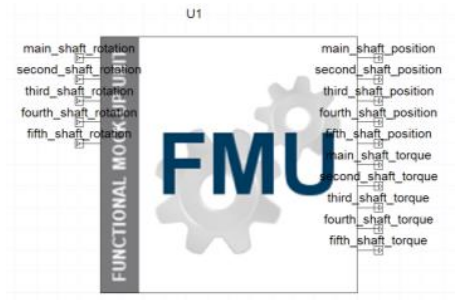

Figure 2 : co-simulation FMU component

It is possible to combine several kinematic models designed separately in the same system assembly, each kinematic model may be converted as a FMU and link to the other in ANSYS Twin Builder.

For the electric motor device, FMUs may also be generated following the same workflow, including also flexible parts such as the housing. It is worth mentioning that when the flexible parts are not undergoing large displacement and rotations, a mechanical State Space model coming from ANSYS finite element solver may also be integrated in this workflow as a model exchange FMU.

\section{System workflows}

The robot model uses 5 motors (one per axis), powered by a circuit mixing voltage sources, MOSFETs and diodes, as shown in figure 3. Each motor provides a rotation angle and get a resistive torque from the FMU pins.

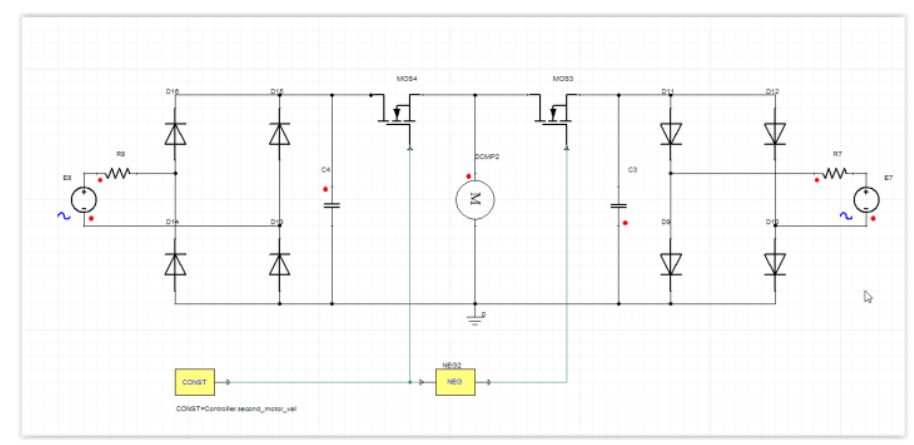

Figure 3 : electric power circuit supplying each robot joint 
To achieve good performance, the kinematic function of the robot, a controller has been defined with SCADE suite and linked to this workflow (figure 4). A target signal has been provided to all electric motors and the system.

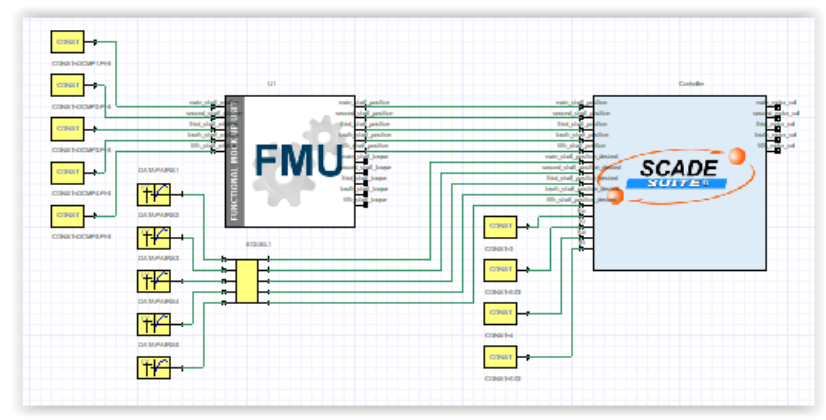

Figure 4 : control of the robot model

Regarding the electric motors, instead of the idealized system blocks (used in figure 3), we may also consider a complex mechanical system involving different FMUs : a rotor with some magnets spinning in a mechanical system will induce some forces/torques on the other mechanical parts, especially on the stator housing which also entails vibrations and unexpected behaviors on the robot arm. Reciprocally, the vibration of the electric motor may be affected by the robot arm connected to this motor and this is also addressed using the co-simulation approach with FMUs. Figure 5 shows the typical combination of FMUs used for the electric motor.

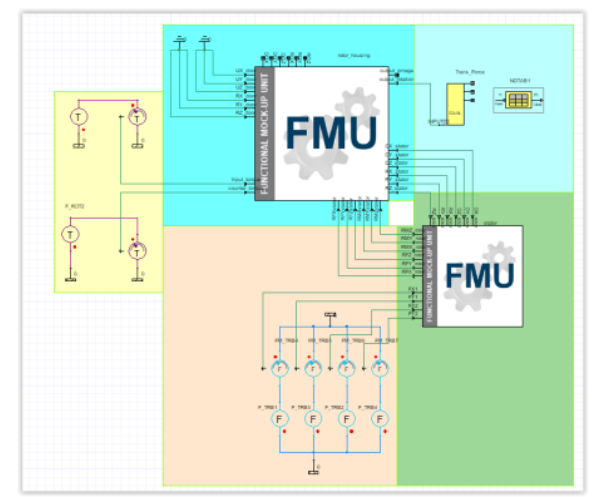

Figure 5 : definition of the workflow for the electric motor

\section{Results}

Figure 6 shows the results at the end of the co-simulation and it emphasizes the behavior of each axis regarding the target signal that the robot arm needs to follow. Here, the controller provides more efficiency for the fourth than for the third axis, especially in terms of response time and smoothness. This may be improved with the use of other components and a smarter controller.
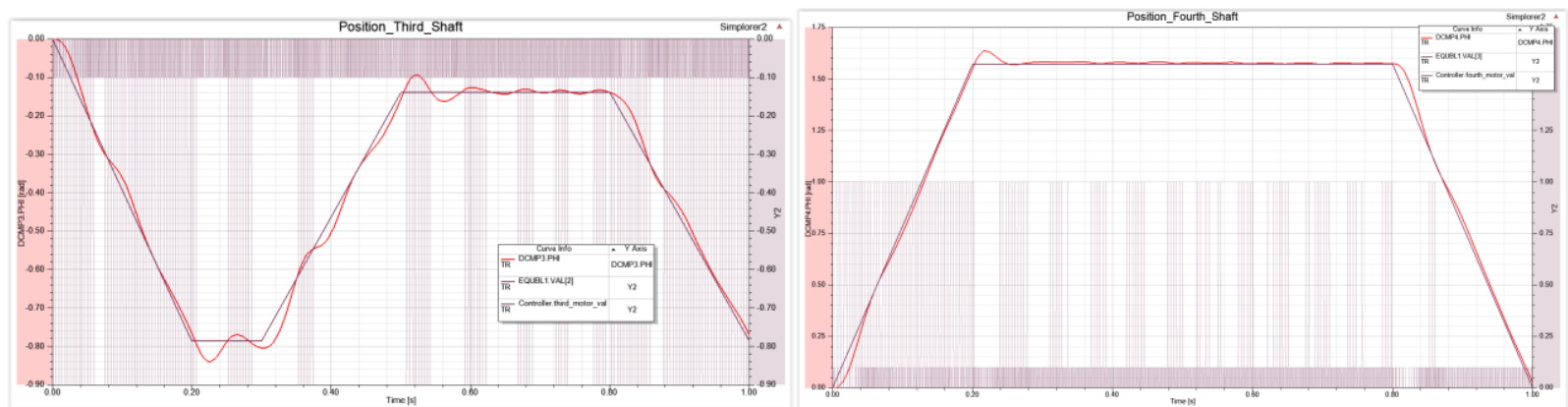

Figure 6 : position results for two different joints of the robot in function of time (s) 
The figure 7 illustrates the difference on the third shaft when we compare the current robot (with one flexible arm) and the same robot with all arms considered as rigid parts. Because of the flexible behavior of arm, the time response of the structure is slightly different, then the controller does not behave exactly in the same way for both cases and we see a small shift between each couple of curves.
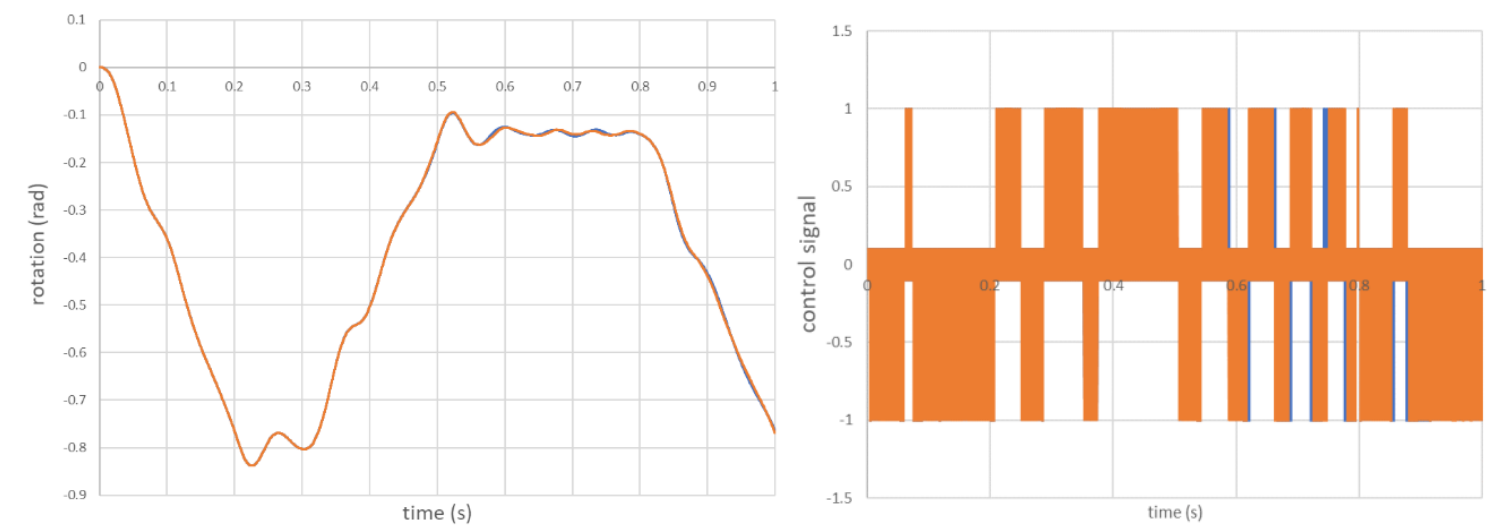

Figure 7: comparison of the position results and the control signal for the full rigid model (orange) and the mixed rigid/flexible (blue) in function of time (s)

\section{Conclusion: Limitations and Extensions}

The FMI standard allows linking together multiple physics without one-off development of coupling interfaces between each of the participant of complex system simulations, thanks to well defined API. Models and Physics that can be easily "reduced" can use the model exchange standard, while highly non-linear system is more subject to use co-simulation FMUs.

While the standard is facilitating exchanges, it also comes with some limitations and difficulties.

- The first of these difficulties lies in validating the produced FMUs. The "Cross Check" tests and the certification procedures are providing some useful information, but it is not sufficient to give confidence on the accuracy and performance of the co-simulation FMUs when coupled to other units.

- Multibody systems, where large rotation usually make it impossible to reduce the model behavior, require cosimulation FMUs. Another source of non-linearities in these systems is collisions and contacts between bodies. Co-simulation becomes in that case more tedious, forcing the synchronization between the participants more frequent due to the non-smooth behavior of the equations of motion [3]. An extension of the standard to events could help handling more efficiently this non-smooth behavior.

- A valuable extension of the standard would be to support full high dimensionality interface, such as a full pressure field on a surface.

\section{References}

[1] fmi-standard.org

[2] Component Mode Synthesis, Jaap Wijker, in Mechanical Vibrations in Spacecraft Design, pp 369-398 Springer, Berlin, Heidelberg

[3] Nonsmooth Mechanics, Models, Dynamics and Control. Third Edition. Bernard Brogliato, Springer, Berlin, Heidelberg 